\title{
No. 394
}

ConocoPhillips and Weatherford Laboratories, Inc. 2011, Geochemical analysis (total organic carbon, rock-eval, permeability to air, porosity, mercury injection capillary pressure, and grain density) from the E. Simpson \#1, Simpson Test Well \#1, North Kalikpik \#1, South Harrison Bay \#1, and Klondike \#1 wells

18 spreadsheets available for download Received November, 2010 